\title{
Reappraisal of Plasmapheresis as a Supportive Measure in a Patient with Hepatic Failure after Major Hepatectomy
}

\author{
Shin Hwang Tae-Yong Ha Chul-Soo Ahn Ki-Hun Kim \\ Sung-Gyu Lee \\ Division of Hepatobiliary Surgery and Liver Transplantation, Department of \\ Surgery, Asan Medical Center, University of Ulsan College of Medicine, Seoul, \\ Korea
}

\section{Key Words}

Plasmapheresis · Hepatectomy · Liver cirrhosis · Hepatic failure

\begin{abstract}
Major resection of cirrhotic livers can result in hepatic failure, but no supportive treatment has been found to be generally effective. We successfully treated a 63-year-old woman with post-hepatectomy liver failure with plasmapheresis. Following right hepatectomy, the initial postoperative recovery of liver function was favorable, except for ascites. One month later, however, the amount of drained ascites increased up to 2 I/day. In addition, serum cholesterol concentration gradually decreased to around 30 $\mathrm{mg} / \mathrm{dl}$, and serum total bilirubin rose to $11.1 \mathrm{mg} / \mathrm{dl}$. Plasmapheresis was performed, and after just 2 sessions, serum cholesterol level was rapidly corrected and prothrombin time was restored. After 3 sessions of plasmapheresis, the usual rebound rise of serum bilirubin disappeared, and the amount of ascites drained also decreased slowly. The patient underwent a total of 5 sessions of plasmapheresis over 2 weeks, after which liver function improved slowly, and she was finally discharged 72 days after liver resection. Mild ascites requiring diuretic therapy persisted over 3 months. She is doing well to date 10 months after liver resection without tumor recurrence or hepatic decompensation. This limited experience suggests that plasmapheresis can be a useful liver support for post-hepatectomy liver failure.
\end{abstract}

\section{Introduction}

Major resection of a cirrhotic liver can result in hepatic decompensation and even fatal hepatic failure. To minimize such operative risk, hepatic reserve is assessed 
preoperatively, including both qualitative and quantitative aspects of liver function. Yet even when these parameters appear to permit liver resection, there is a non-negligible possibility of post-hepatectomy liver failure (PHLF) $[1,2]$. Once PHLF occurs after liver resection, there are few treatment modalities, except for general liver supports, to avoid further aggravation.

We recently treated a patient with PHLF after right hepatectomy, whose liver function recovered following plasmapheresis although the latter was known to have only limited and transient effects. Since the clinical sequence of this patient showed the beneficial effects of plasmapheresis on PHLF, we describe it in detail.

\section{Case Report}

The patient was a 63-year-old woman with a hepatocellular carcinoma in the right lobe and a background of hepatitis B-associated liver cirrhosis. She had suffered from a cerebral infarct 10 years earlier, but recovered completely without sequelae. A 5-cm sized single nodular hepatocellular carcinoma in the segment VII was detected during a routine checkup (fig. 1a). Computed tomography (CT) volumetry showed that the volume of the left lobe, including the caudate lobe, was equivalent to $45 \%$ of the whole liver volume. The 15 -minute retention rate of indocyanine green was $8.8 \%$, and serum biochemistry included aspartate aminotransferase (AST) $124 \mathrm{IU} / \mathrm{l}$; alanine aminotransferase (ALT) 28 IU/l; total bilirubin $0.7 \mathrm{mg} / \mathrm{dl}$; albumin $3.5 \mathrm{~g} / \mathrm{dl}$; and prothrombin time $99 \%$ (1.0 of international normalization ratio [INR]).

Although these liver profiles appeared permissible for right lobectomy, we planned to perform percutaneous transhepatic portal embolization (PTPE) to minimize the risk of surgery, due to this patient's liver cirrhosis and splenomegaly. During PTPE, main portal vein pressure became rather high, being $17 \mathrm{~mm} \mathrm{Hg}$ before and $24 \mathrm{~mm} \mathrm{Hg}$ after embolization of the right portal vein. Her liver function remained stable after PTPE, and CT performed 2 weeks later showed that left lobe volume was $51 \%$ of the whole liver volume (fig. 1b), indicating that $6 \%$ of the whole liver volume had shifted. Endoscopic examination showed no evidence of gastroesophageal varices.

Right lobectomy was performed 20 days after PTPE. The right lobe showed noticeable wrinkling at the cirrhotic background, which made the interlobar demarcation noticeable. During surgery, the right hepatic pedicle was initially looped using an extraglissonian approach method after full mobilization of the right liver. Following simultaneous clamping of the right glissonian pedicle and right hepatic vein as a hemi-hepatic block, the liver parenchyma was transected with a Cavitron ultrasonic surgical aspirator and electrocautery. Inflow to the left lobe was not interrupted at all. Intraoperative blood loss was about $300 \mathrm{ml}$, and the patient did not require transfusion.

During the first week after right lobectomy, the worst laboratory values were AST 124 IU/l, ALT 33 $\mathrm{IU} / \mathrm{l}$, total bilirubin $2.1 \mathrm{mg} / \mathrm{dl}$, albumin $2.6 \mathrm{~g} / \mathrm{dl}$, and prothrombin time $57 \%$ (1.31 INR). Intravenous hyperalimentation was performed for 5 days, and oral intake was encouraged [3]. There was no evidence of surgical complication, but ascites drainage from an abdominal drain persisted at 1-2 1/day despite apparent recovery of liver function (fig. 1c). After 1 month, the amount of drained ascites did not decrease in spite of drain clamping, and serum cholesterol level gradually decreased to around 30 $\mathrm{mg} / \mathrm{dl}$, despite vigorous hyperalimentation and oral intake (fig. 2). At that time, serum total bilirubin began to rise and prothrombin time became significantly prolonged (fig. 2). The serum ammonia concentration exceeded $100 \mu \mathrm{mol} / \mathrm{l}$, but overt hepatic encephalopathy did not develop. There was no evidence of infection, including bacterial peritonitis. Serial CT follow-up showed that the remnant left lobe had fully regenerated and filled the upper abdomen (fig. 1d).

The production of a massive amount of ascites persisted and serum total bilirubin increased progressively to $11.1 \mathrm{mg} / \mathrm{dl} 48$ days after right lobectomy. Plasmapheresis was started after insertion of a large-bored catheter into the internal jugular vein. After just 2 sessions of plasmapheresis, serum cholesterol level was rapidly corrected and prothrombin time was restored. These beneficial effects persisted during the 2-3 day intervals between plasmapheresis sessions. After 3 sessions of plasmapheresis, the usual rebound increase in serum bilirubin after plasmapheresis disappeared, and the amount of draining ascites also decreased (fig. 2).

This patient underwent a total of 5 sessions of plasmapheresis over 2 weeks, after which the peritoneal drain was successfully removed. Her liver function improved slowly thereafter, and she was 
finally discharged 72 days after right lobectomy. During monthly follow-up visits at the outpatient clinic, she showed mild ascites retention for the first 3 months. Ten months later, she is doing well, without tumor recurrence or deterioration of liver function.

\section{Discussion}

Progressive deterioration of liver function after resection of a cirrhotic liver may lead to a fatal sequence if not corrected in time. To reverse the sequence of PHLF, liver function support during the acute phase of deterioration appears critical. Various blood purification therapies have been developed for treatment of liver failure [4]. Due to largevolume liver transplantation in our institution, we have often performed plasmapheresis and molecular adsorbent recirculating system (MARS), but its beneficial effects appeared very limited and transient in patients with serious liver failure or graft dysfunction [5-7].

Plasmapheresis, a method of plasma exchange to replace fresh plasma after separation from blood, has had encouraging results in selected patients $[6,8]$. Soon after plasmapheresis, two important biochemical parameters were corrected in our patient. The first was restoration of serum cholesterol level. Although we initially suspected that this was due to replacement by the cholesterol content in the donor plasma, we found that, after only 2 sessions of plasmapheresis, the cholesterol concentration remained over $100 \mathrm{mg} / \mathrm{dl}$ during the interval between sessions. Hypocholesterolemia has been found to be an independent predictor of mortality in critical surgical illnesses $[9,10]$. In our patient, the restoration of serum cholesterol level was thought to reflect the sequence of recovery of liver function. Temporary liver support using plasma exchange may trigger the functional recovery of a failing liver. The second important biochemical parameter improved after plasmapheresis was prothrombin time, which showed a similar pattern of recovery as serum cholesterol level. Initial improvement of prothrombin time may have been due to coagulation factor replacement, but its recovery pattern was indicative of the rapid recovery of hepatic synthetic function.

In contrast to serum cholesterol and prothrombin time, the decrease in serum bilirubin concentration was rather delayed. We had to continue plasmapheresis until the disappearance of the rebound rise of serum bilirubin, because the initial, temporary decease in bilirubin concentration was due to blood dilution.

The recovery sequence in this patient can be summarized as follows: After initial recovery from right lobectomy, excessive ascites production from portal hypertension and liver cirrhosis induced a nutritional disturbance, followed by decreases in hepatic synthetic and metabolic function. After plasmapheresis, the intrinsic cholesterol pool was first restored, followed by improvements in hepatic synthetic and metabolic function; after restoration of overall liver function, ascites finally decreased. Based on this reasoning, plasmapheresis for PHLF is indicated for only a limited number of patients, because it is only a temporary supportive therapy. Although liver support from plasmapheresis must therefore be too weak to reverse the sequence of definite hepatic failure, our experience with this patient suggests that, in patients with failing liver function, even the slight supportive effect provided by plasmapheresis can trigger spontaneous recovery.

In conclusion, this limited experience suggests that plasmapheresis can be a useful liver support for PHLF. 
Fig. 1. Sequential CT scans in our patient. a Initial CT scan showing a nodular hepatocellular carcinoma mass in segment VII. b CT scan 2 weeks after PTPE, showing slight regeneration of the left liver parenchyma. c CT scan 1 week after right lobectomy, showing fluid collection around the evacuated right subphrenic space. d CT scan 6 weeks after right lobectomy, showing full regeneration of the remnant left lobe. Intraabdominal ascites collection was decreased after continuous external drainage.
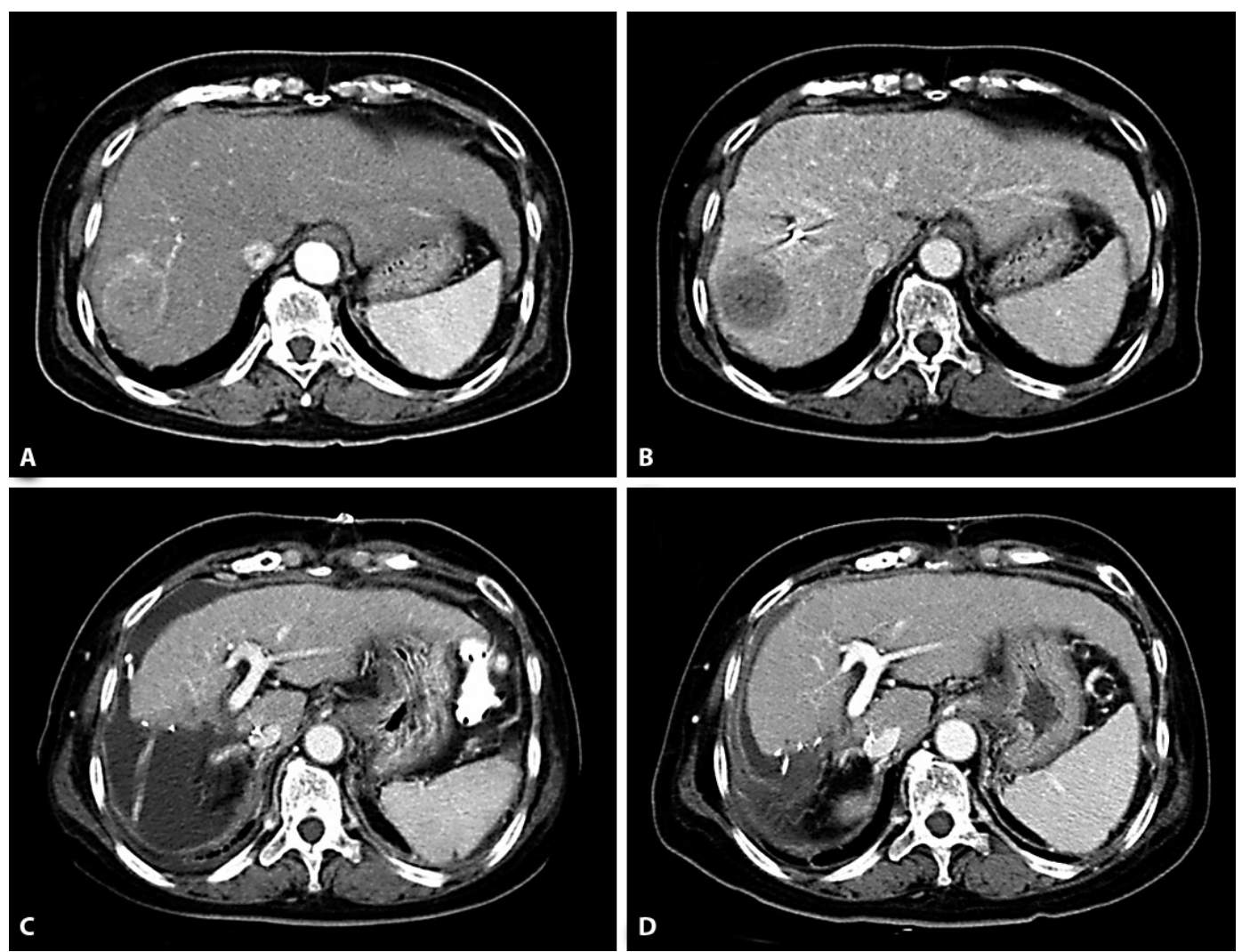
Fig. 2. Sequential changes in daily drained ascites through the external drainage tubes and concurrent changes in liver functional profiles. A blank arrow indicates the attempts of abdominal drain clamping and solid arrows indicate the sessions of plasmapheresis.
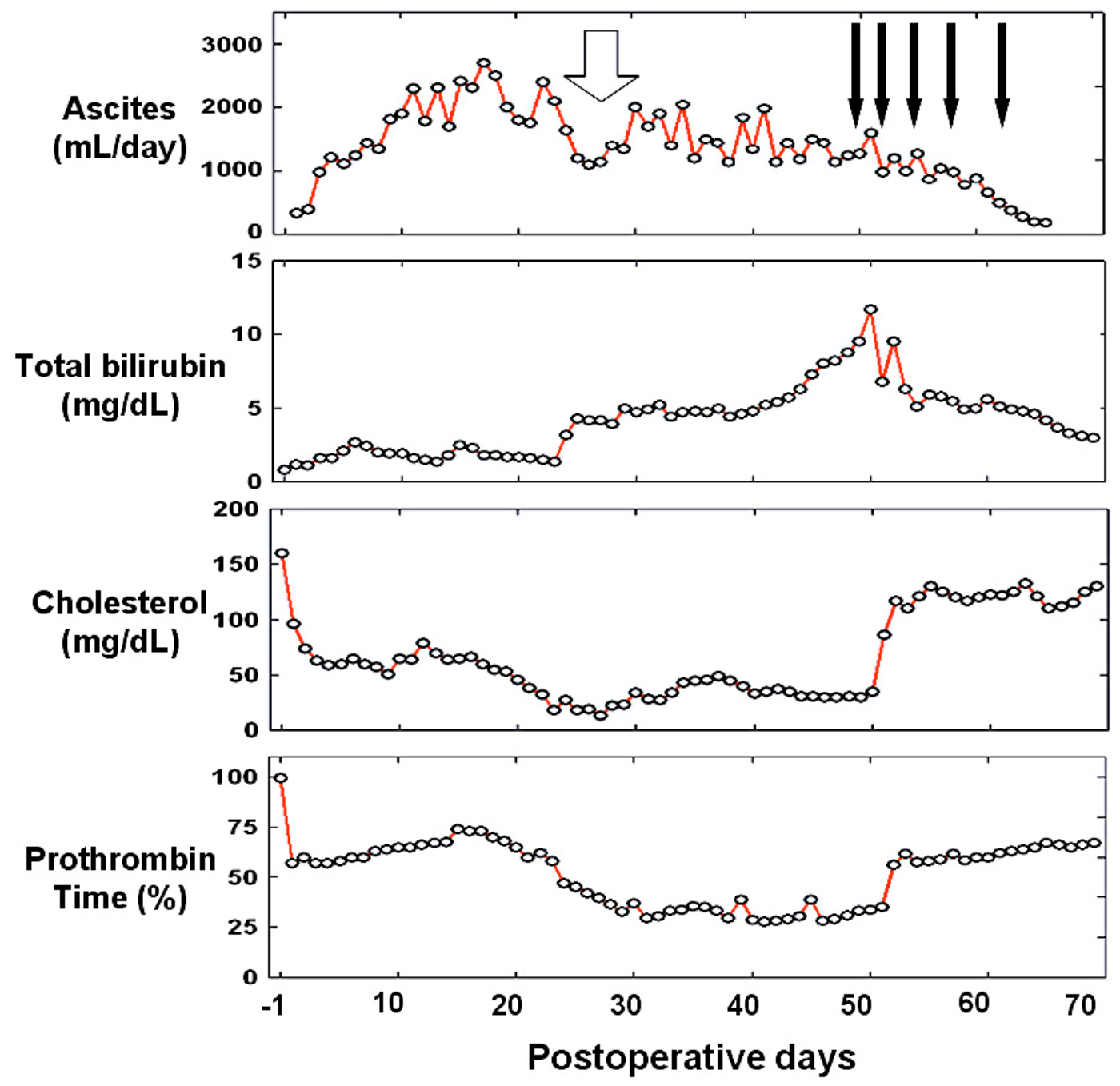


\section{References}

1 Lee SG, Hwang S: How I do it: assessment of hepatic functional reserve for indication of hepatic resection. J Hepatobiliary Pancreat Surg 2005;12:38-43.

2 Shirabe K, Shimada M, Gion T, Hasegawa H, Takenaka K, Utsunomiya T, Sugimachi K: Postoperative liver failure after major hepatic resection for hepatocellular carcinoma in the modern era with special reference to remnant liver volume. J Am Coll Surg 1999;188:304-309.

-3 Richter B, Schmandra TC, Golling M, Bechstein WO: Nutritional support after open liver resection: a systematic review. Dig Surg 2006;23:139-145.

-4 Naruse K, Nagashima H, Sakai Y, Kokudo N, Makuuchi M: Development and perspectives of perfusion treatment for liver failure. Surg Today 2005;35:507-517.

5 Hwang S, Lee SG, Lee YJ, Sung KB, Park KM, Kim KH, Ahn CS, Moon DB, Hwang GS, Kim KM, Ha TY, Kim DS, Jung JP, Song GW: Lessons learned from 1,000 living donor liver transplantations in a single center: how to make living donations safe. Liver Transpl 2006;12:920-927.

6 Mandal AK, King KE, Humphreys SL, Maley WR, Burdick JF, Klein AS: Plasmapheresis: an effective therapy for primary allograft nonfunction after liver transplantation. Transplantation 2000;70:216-220.

7 Chiu A, Chan LM, Fan ST: Molecular adsorbent recirculating system treatment for patients with liver failure: the Hong Kong experience. Liver Int 2006;26:695702 .

-8 Ozdemir FN, Tutal E, Sezer S, Gur G, Bilgic A, Haberal M: Effect of supportive extracorporeal treatment in liver transplantation recipients and advanced liver failure patients. Hemodial Int 2006; 10:S28-S32.

9 Gui D, Spada PL, DeGaetano A, Pacelli F: Hypocholesterolemia and risk of death in the critically ill surgical patient. Intensive Care Med 1996;22:790-794.

10 Giovannini I, Boldrini G, Chiarla C, Giuliante F, Vellone M, Nuzzo G: Pathophysiologic correlates of hypocholesterolemia in critically ill surgical patients. Intensive Care Med 1999;25:748-751. 